\title{
Title: DNA methyltransferases and gastric cancer: insight into targeted therapy
}

Short running title: DNA methyltransferases and targeted therapy

Sadegh Fattahi ${ }^{1,5}$, Monireh Golpour ${ }^{2}$, Fatemeh Amjadi-Moheb ${ }^{3}$, Marzieh Sharifi-Pasandi ${ }^{2}$, Parastesh Khodadadi $^{3}$, Maryam Pilehchian-Langroudi ${ }^{3}$, Hossein Ashrafi ${ }^{4}$, Haleh Akhavan-Niaki ${ }^{3,5^{*}}$

1- Cellular and Molecular Biology Research Center, Health Research Institute, Babol University of Medical Sciences, Babol, Iran

2- Molecular and Cell Biology Research Center, Student Research Committee, Faculty of Medicine, Mazandaran University of Medical Science, Sari, Iran

3- Department of Genetics, Faculty of Medicine, Babol University of Medical Sciences, Babol,

$$
\text { Iran }
$$

4- Kingston University London, Cancer theme, School of Life Science, Pharmacy and Chemistry,

SEC Faculty, Kingston upon Thames, KT12EE, London, UK

5- North Research Center, Pasteur Institute, Amol, Iran

\section{Corresponding author details:}

Haleh Akhavan-Niaki, Department of Genetics, Faculty of Medicine, Babol University of Medical Sciences, Babol, Iran. Phone: 0098911125 5920, Fax: 0098-114 319 8067, Email: halehakhavan@yahoo.com (please note only one author can be listed as corresponding author).

Financial disclosure: N/A

Acknowledgements: N/A

Information pertaining to writing assistance: N/A

Ethical disclosure: N/A

Author Contributions:

Word count:

Figure number: 6

Table number: 3 


\title{
DNA methyltransferases and gastric cancer: insight into targeted therapy
}

\begin{abstract}
Gastric cancer is a major health problem worldwide occupying most frequent causes of cancerrelated mortality. In addition to genetic modifications, epigenetic alterations catalyzed by DNA methyltransferases (DNMTs) is a well-characterized epigenetic hallmark in gastric cancer. The reversible nature of epigenetic alterations and central role of DNA methylation in diverse biological processes provides an opportunity for using DNA methyltransferase inhibitors to enhance the efficacy of chemotherapeutics. In this review, we discussed key factors or mechanisms such as SNPs, infections, and genetic modifications that trigger DNMTs level modification in gastric cancer, and their potential roles in cancer progression. Finally, we focused on how inhibitors of the DNMTs can most effectively be used for the treatment of gastric cancer with multidrug resistance.
\end{abstract}

Keywords: DNA methyltransferase (DNMT); epigenetic alterations; gastric cancer; drug resistance; chemotherapy

\section{Introduction}

Gastric cancer is caused in part by genetic and epigenetic alterations in oncogenes and tumor suppressor genes (TSGs) [1]. Although epigenetic modifications are regulators and natural phenomenon occurring during normal development, tissue-specific gene expression, and cell functions; aberrant epigenetic modifications can have harmful effects that can contribute to cancer development [2-3]. New and ongoing researches are continuously uncovering the interaction between genes and environment through epigenetic alterations which make a person 
susceptible to develop gastric cancer [4-5]. Aberrant epigenetic modifications can dysregulate the transcription level of TSGs and oncogenes through different mechanisms, without any changes in DNA nucleotide sequences of the genes [1]. Epigenetic alterations including histone modification, non-coding RNA, and DNA methylation may initiate and sustain changes which lead to the inactivation of tumor suppressor and other cancer-related genes in gastric cancer [68]. Histone proteins determine the chromatin structure and function through a variety of modifications like acetylation and methylation in their $\mathrm{N}$-terminal domain. These modifications have effects on a number of pivotal molecular processes including gene transcription, DNA replication, and nucleosome positioning. Depending on the type of histone modification, transcription is either activated or repressed. Histone hyper-acetylation is linked with transcriptional activation, but the outcome of histone methylation relies completely on the type of modified amino acid residue, and the degree of methylation. For instance, trimethylation of $\mathrm{H} 3 \mathrm{~K} 4$ may promote gene expression, while trimethylation of $\mathrm{H} 3 \mathrm{~K} 9$ and H3K27 suppresses gene expression. Interestingly, tumor suppressive and oncogenic miRNAs are well-known epigenetic hallmarks of gastric cancers [3]. One third of miRNAs dysregulation is associated with histone modifications and hypo/hypermethylation of $\mathrm{CpG}$ islands which are located in their promoter and 5' regulatory end regions. MiRNAs bind to the 3' untranslated region of their target genes and cause post-transcriptional gene inactivation [9]. Among the epigenetic processes, DNA methylation is a fundamental mechanism which plays a pivotal role in biological functions responsible for DNA stability preservation such as genomic imprinting, and X-chromosome inactivation. It is not unexpected that aberrant DNA methylation induce the development of numerous malignancies, especially gastric cancer [10-12]. DNA methylation in gastric epithelia can also be influenced by several factors including age, diet, physical activity, chronic inflammation, and infectious agents [13]. Global DNA hypo/hyper methylation frequently occur in gastric tumors, which make contribution to genomic instability 
$[10,12]$. In fact, aberrant hyperactivation of DNA methyl transferases (DNMTs) is responsible for TSGs silencing or inactivation, and eventually gastric cancer development [14-15].

\section{DNA methyltransferases classification}

Based on their structure, DNMTs are composed of three main types, DNMT1, DNMT2, and DNMT3. Maintenance methyltransferase (DNMT1) and de novo methyltransferases (DNMT3a, DNMT3b, and DNMT3L) are two major enzyme types with $\mathrm{m}^{5} \mathrm{C}$ methyltransferase activity (C-5 cytosine-specific DNA methylase or C5 Mtase), which trigger the transfer of a methyl group to C-5 carbon of cytosines in mammalian DNA [16-17]. DNMT1 is responsible for maintenance of DNA methylation status of newly synthesized daughter strands during replication, leading to full DNA strands methylation. DNMT3 consists of three subtypes comprising DNMT3a, DNMT3b, and DNMT3-Like protein (DNMT3L). DNMT3a and DNMT3b contribute to catalyze DNA methylation pattern throughout the nucleus without distinguishing hemi-methylated from methylated $\mathrm{CpG}$ sites during embryogenesis and germ cell development. DNMT3L is known as a regulatory protein for de novo methylation. It has been shown that DNMT3L participates in regulating the DNMT3a and DNMT3b activity for de novo methylation. DNMT2 is known as a DNA methyltransferase which lacks regulatory domain, but new findings showed the implication of this enzyme in adding methyl groups to tRNA anti-codon loop [18]. In general, the DNMTs consist of a C-terminal catalytic domain and an N-terminal regulatory domain (Fig. 1). The catalytic domain is conserved between DNMTs while the regulatory domain is variable in size and amino acid sequence. These two domains are linked by glycine-lysine repeats (GK)n. The catalytic domain in DNMTs consists of six motifs including I, IV, VI, VIII, IX and X. Motifs I and X are S-adenosylmethionine (SAM) binding site while motifs VIII and IX are implicated in DNA binding. DNMT3L lacks motifs IX and $\mathrm{X}$ in its catalytic domain. The $\mathrm{N}$-terminal regulatory region of DNMT1 is 
composed of several domains that are DNA methyltransferase associated proteins (DMAP); Proliferating cell nuclear antigen (PCNA) binding domain (PBD), nuclear localization signal (NLS), replication foci targeting sequence (RFTS), cysteine rich, zinc finger DNA-binding motif (CXXC), and bromo-adjacent homology Dmap1-binding region (BAH1 and BAH2). DMAP1 domain is responsible for binding of DNMT1 with the transcriptional repressor DMAP1 [19]. The PBD domain is able to interact with PCNA, and recruits DNMT to the replication foci in S phase. Relatively, the absence of PBD domain delays the methylation after replication [20]. RFTS domain is responsible for replication-coupled DNA methylation at the differentially methylated regions of imprinted genes through binding to SRA domain of UHRF1 (ubiquitin like with PHD and ring finger domains 1) protein that is a replicationcoupled methylation factor. In addition, RFTS domain protects the genome against aberrant methylation. It was reported that, truncated DNMT1 which lacks parts of the RFTS domain is unable to perform replication-coupled DNA methylation at the differentially methylated regions of imprinted genes, and can trigger global methylation of the genome as well [21-22]. The CXXC domain binds to unmethylated $\mathrm{CpG}$ dinucleotides, and is crucial for DNMT1 enzymatic activity [23]. Both BAH motifs in PBHD domain are essential for the folding of DNMT1 [24-25]. Structurally, the N-terminal region of DNMT3 contains a variable region (280 amino acids in DNMT3a, and 220 amino acids in DNMT3b), and two conserved PWWP (tetrapeptide domain containing proline-tryptophan-tryptophan-proline motif) domains and the ATRX-Dnmt3-Dnmt3L (ADD) domain [26-27]. The PWWP domain that contains 100-150 amino acids is conserved in both DNMT3a and DNMT3b [25]. This domain is responsible for the methylation of major satellite repeats at pericentromeric chromatin, and recognizes the H3K36me3 mark [28-29]. The ADD domain is composed of three cysteine-rich subdomains, and recognizes the unmethylated state of lysine 4 and 9 in histone H3 [30-32]. 


\section{Targets of DNMT in gastric cancer}

Aberrant DNA methylation due to the DNMTs up regulation may trigger tumor progression, invasion, and metastasis through down regulation of genes that have a role in proliferation inhibition and apoptosis-related pathway [33]. Several studies indicate that aberrant expression of DNMTs is closely linked to hypermethylation of TSGs. A series of genes which may become hypermethylated during gastric cancer development are listed in table 1. Many of these genes are involved in cell cycle regulation, apoptosis, proliferation, migration and major cell signaling pathways such as epithelial mesenchymal transition (EMT) ), notch, Wnt/ $\beta$ catenin, and AKT/mTOR. Investigations suggested that deregulation of these types of genes would significantly affect cancer progression. Wnt/ $\beta$-catenin signaling pathway has a fundamental role in controlling the cellular processes such as cell proliferation, cell cycle control, and migration of epithelial cells of gastric mucosa. Alteration of Wnt signaling has been found to be implicated in gastric cancer development. Hypermethylation of Wnt related inhibitor genes including $s F R P 1, s F R P 2, s F R P 4, s F R P 5, D K K-1, D K K-2, D K K-3, S O X 10$, SOX17, WIF-1, NKD1, HSULF-1, RUNX3, PRDM5, RASSF10, OSR1, and APC at the CpG island contribute to tumor progression through Wnt pathway activation [34-35]. It is known that AKT/mTOR pathway together with other signaling pathways acts as a crucial regulator of cell growth, metabolism, apoptosis, metastasis, and angiogenesis [36]. The alteration of AKT/mTOR pathway is associated with gastric tumor progression. Methylation of the TSGs that are responsible for inhibition of AKT signaling can lead to AKT activation and apoptosis evasion in tumor cells. Phosphatase and tensin homolog (PTEN) and A disintegrin-like and metalloprotease with thrombospondin type 1 motif, 9 (ADAMTS9) which act as suppressors for AKT pathway have been indicated to be silenced in gastric cancer [37-39]. Moreover, it 
has been reported that notch signaling is a conserved pathway that plays a critical role in cellular functions vital for cell survival. Notch may act as both an oncogene and a suppressor in gastric cancer [40]. Epigenetic alterations of this pathway may lead to gastric cancer progression. Studies in gastric cancer cell lines demonstrate that promotor methylation of deltalike canonical notch ligand 1 (DLL1) which encodes for a ligand of NOTCH1 was restored following treatment with 5-Aza-2'-deoxycytidine (5-Aza-dC), a DNMTs inhibitor [41-42]. Conversely, other investigations show that NOTCH1 can promote tumorigenesis by cyclooxygenase-2 activation [43]. It seems that the function of notch pathway components is ambiguous as they were reported either as suppressor or activator of gastric carcinoma, and their exact effect on different steps of gastric cancer pathogenesis still remains unclear [40, 42, 44-45]. Iroquois homeobox 1 (IRX1) is a member of Iroquois homeobox factors family that is required for embryonic development [46]. Down-regulation of IRXI has been reported in several cancers including gastric cancer. Guo et al. have shown that exposure with 5-Aza-dC could restitute IRX1 protein level in gastric cancer cells [47]. Reprimo (RPRM) has been reported to be inhibited by DNMTs and its function was restored by treatment with zebularine, a DNMT inhibitor [48]. Moreover, a possible association between DNMT1 amplification, triggering hypermethylation of $\mathrm{CpG}$ islands and down-regulation of TSGs including human MutL homolog 1 (hMLH1), thrombospondin 1 (THBS1), cadherin 1 ( CDH1) has been suggested in gastric cancer [49]. Besides, due to adverse effects of chemical drugs, DNMTtargeted inhibition with small interfering ribonucleic acids (siRNAs) for reactivation of silenced genes including cyclin-dependent kinase inhibitor 2A (CDKN2A), A-kinase anchoring protein $12(A K A P 12 B)$, runt related transcription factor $3(R U N X 3)$, helicase-like transcription factor $(H T L F)$, and ras association domain family member $1(R A S S F 1 A)$ was used as a potential therapeutic strategy [50]. 


\section{Expression of DNMTs and susceptibility to infections in gastric cancer}

Although some clinical evidence show the elevated expression of DNMTs in gastric cancer, and consequent silencing of TSGs during tumorigenesis, little is known about the mechanisms that trigger or cause aberrant methylations [14-15]. APC is a TSG that was introduced as a gate keeper, and mutation in this gene plays an important role in gastric cancer as well as in colorectal carcinogenesis [100]. The role of APC in regulating DNMTs level was examined in colorectal cancer, and it was found that full-length APC but not truncated APC is able to suppress DNMT1 activity [101]. Mutations in $A P C$ gene lead to the production of truncated protein which cannot suppress DNMT1 expression, and may be responsible for DNMT1 upregulation in gastric cancer [100]. It has been also reported that exposure of gastric cancer cells to nitric oxide (NO) produced induced DNA methylation by enhancing DNMT enzymatic activity [102-103]. Furthermore, infectious agents can induce epigenetic modifications, and be the most common complications in gastric cancer [104-105]. Infections hijack the host DNA methylation mechanism via increasing DNMTs in order to control host transcription to their benefit. Figure 2 summarizes different ways through which infections increase DNMTs expression in gastric cancer. Helicobacter pylori and Epstein-Barr virus (EBV) are wellknown carcinogenic risk factors in gastric cancer that facilitate DNMTs function and hypermethylation of $\mathrm{CpG}$ islands of various cancer-associated genes. Furthermore, several studies have indicated the presence of HPV (Human papillomavirus) in patients with gastric cancer, suggesting its potential role as risk factor for gastric cancer [106-107]. Study on human cervical carcinoma SiHa and CaSki cell lines has shown that P53 TSG suppresses DNMT1 expression [108]. DNMT1 was also transcriptionally suppressed by P53 in non-small cell lung cancer (NSCLC) patients and A549 cell lines. P53 could repress DNMT1 expression by 
interacting with SP1. This complex binds to both P53 and SP1 putative binding sites on DNMT1 promoter. Unlike P53, SP1 can lead to increased DNMT1 expression. Thus, mutation in P53 and overexpression of SP1 can trigger DNMT1 mediated hypermethylation of TSGs promoters [109]. Study on cervical cancer has shown that HPV-16 E6 protein probably upregulates DNMT1 expression by repressing P53 [108]. In addition to P53, RB could negatively regulate $D N M T$ s expression through interaction with DNMT3a. Murine double minute 2 (MDM2) which encodes a nuclear-localized E3 ubiquitin ligase targets both P53 and $\mathrm{RB}$, and therefore causes overexpression of DNMTs in cancer cells [110]. Infections increase the DNMTs expression by secreting proteins able to activate various signaling pathways [111]. In addition, chronic inflammation which occurs in the presence of infections in cancer cells promotes differentiation and polarization of monocytes into tumor-associated macrophages (TAMs). TAMs, as abundant inflammatory cells in tumor microenvironment, increase DNMT1 expression by secreting chemokine (C-C motif) ligand 5 (CCL5) [112]. CCL5 a known biomarker in late stage of gastric cancer is upregulated in cells infected by $H$. pylori, EBV, and HPV [113-117]. Therefore, by inducing CCL5 chemokine, these infections may upregulate DNMTs in gastric cancer tissue. H. pylori infection is the main factor that causes gastric inflammation, and promotes aberrant DNA methylation. DNMT3a was indicated as a poor prognostic hallmark for gastric cancer infected with $H$. pylori [118]. Induction of AKT phosphorylation as the main contributor in AKT-NFאB pathway by $H$. pylori can stimulate DNMTs overexpression leading to TSGs hyper-methylation. Promoter hyper-methylation of TSGs is important in tumorigenesis. CagA the important gene of $H$. pylori, could increase AKT phosphorylation by activated PDK1. Phosphorylated AKT (P-AKT) induces DNMT1 expression by $N F \kappa \beta$ activation. Therefore, $N F \kappa \beta$ could play a role in up-regulating the expression of DNMT by binding directly to its promoter [111]. H. pylori infection can also induce IL-1 $\beta$ as an important pro-inflammatory cytokine that has considerable function in 
initiating and amplifying the inflammatory response. Increased IL-1 $\beta$ is followed by DNMT induction by activated inducible nitric oxide synthase (iNOS) and NO production which have an important role in aberrant DNA methylation and gastric carcinogenesis [103, 119-120]. $H$. pylori infection may directly effect on gastric cells by inducing macrophages activation, which causes an increase of NO production in gastric cells. Overproduction of NO can activate DNMTs to promote DNA methylation [121]. H. pylori infection through increased expression of IL-6 and IL-11 can activate signal transducer and activator of transcription 3 (STAT3) [122]. STAT3 can affect gene expression through epigenetic changes including DNA methylation and chromatin modulation. This transcription factor increases $\mathrm{CpG}$ island methylation of TSGs through interacting with DNMT1 [123].

Interestingly, aberrant DNA methylation is strongly induced even in normal tissues by exposure to chronic inflammation due to $H$. pylori infection. Maekita et al. found that some $\mathrm{CpG}$ islands regions were significantly highly methylated in gastric mucosae of $H$. pylori-infected individuals in comparison with non-infected individuals [124]. Moreover, methylation levels of three tumor-suppressor miRNAs were found to increase in non-cancerous individuals infected with H. pylori in comparison with noninfected healthy subjects [125]. Furthermore, in vivo study indicated that inflammations triggered by $H$. pylori infection has a strong potential to induce aberrant DNA methylation in gastric epithelial cells [126]. Inflammation due to $H$. pylori infection induced the expression of chemokine (C-X-C motif) ligand 2 (CXCL2), IL-1b,NOS2, and tumor necrosis factor-alpha (TNF- $\alpha)$ genes, in parallel to DNA methylation [126-127]. Accumulation of aberrant DNA methylation in gastric epithelial cells due to inflammatory response associated with $H$. pylori infection may favor cancerogenesis [126].

In addition, TAMs can induce DNMT1 expression through CCL5/CCR5/STAT3 signaling pathway in gastric cancer cells. Overexpression of DNMT1 mediated by TAMs also induces gelsolin silencing and may lead therefore to gastric cancer progression [128-131]. The molecular mechanism of aberrant DNA methylation caused by EBV infection is not still clear. 
However, at least 2 mechanisms by which EBV can effect DNA methylation have been proposed. One mechanism is that viral latent membrane protein 2A (LMP2A) can increase the phosphorylation of STAT3, which up regulates the DNMT1 and DNMT3b expression and cause epigenetic changes in gastric cells [132-133]. Also, EBV can induce DNMT1 expression through the viral oncoprotein LMP1 via JNK/AP1-signaling. LMP1 has two main regions called C-terminal activating region (CTAR1 and CTAR2). The YYD domain in CTAR2 can play an important role in activating DNMT1 gene expression. The YYD domain activates JNK kinase and upon phosphorylation of c-Jun, in turn c-Jun binds to AP1 site of DNMT1 promoter [134].

\section{Polymorphisms in DNMTs and gastric cancer development}

Accumulating evidence demonstrate that genetic variations of DNMTs particularly single nucleotide polymorphisms (SNPs), and their haplotype blocks are associated with the occurrence rate of many cancers including gastric cancer [135]. SNPs may be responsible for promoter activity alteration, gene expression modulation, splice site change, transcription factor binding site change, and epigenetic modification [136]. Conspicuously, finding relevant polymorphisms can be used as a potential biomarker for gastric cancer prediction. Wang et al. indicated that the GG genotype of DNMT3A rs1550117 variant decreased the death risk of gastric cancer, and may therefore be a potential prognostic marker in gastric cancer [137]. Many studies have described the association of two common polymorphisms rs1569686 ($579 \mathrm{G}>\mathrm{T})$ and $\mathrm{rs} 2424913(-149 \mathrm{C}>\mathrm{T})$ on the promoter of $D N M T 3 B$ which alter the promoter activity [138-139]. However, there is conflicting evidence on the contribution of these two SNPs in different cancers [140-143]. Hu et al. [144] and Chen et al. [145] reported the association of rs1569686 with gastric cancer risk in the Chinese population, while Wang et al. [146] and Ahmadi et al. [147] did not observe any significant association in the Chinese and 
Iranian population, respectively. Likewise, other studies demonstrated that rs2424913 was irrelevant to the risk of gastric carcinogenesis [144-145, 148-150]. Moreover, haplotype analysis showed that a haplotype block between rs1569686 and rs2424913 in DNMT3B locus [145] which carries -149T/-579T increased the susceptibility to gastric cancer. However, few other studies have shown no significant association between DNMTs variants and the risk of gastric cancer development. Table 2 summarizes the associated and non-associated variants of DNMTs with the risk of gastric carcinogenesis. Some studies have found that DNMTs variants can alter the survival rate of gastric cancer. Correspondingly, TG/GG genotypes of DNMT3B rs1569686 and AG/AA genotypes of DNMT3A rs1550117 were associated with poor survival of gastric cancer [137, 146], while GA/AA genotypes of DNMT1 rs2228611 were associated with higher rates of gastric cancer survival [151]. The effect of $H$. pylori infection as the main cause of gastric atrophy and gastric cancer, depends on host characteristics such as SNPs of DMNTs [152]. However, few studies have investigated the correlation between $H$. pylori infection with DNMTs SNPs in gastric cancer occurrence. Relatively, Jiang et al. [152] studied DNMT1 polymorphisms and demonstrated that the AA genotype of rs2228349 had higher risk of $H$. pylori infection whereas, the GG genotype of rs10420321 and the CC genotype of rs 8111085 had lower risk of $H$. pylori infection but a higher risk for gastric atrophy susceptibility [152]. Furthermore, Cao et al. [153] found that the AA genotype of rs1550117 in DNMT3A had higher risk of $H$. pylori infection, but they did not observe any association of this SNP with gastric cancer and gastric atrophy. Among the studied SNPs, the 3'UTR SNPs can be recognized by miRNAs. Nevertheless, the key impact of miRNAs on posttranscriptional regulation of gastric cancer regulator genes via binding with their target sites on 3'UTR, and the influence of polymorphisms in miRNAs target sites haven't been studied yet. Therefore, we looked for miRNA target sites alteration through studied DNMTs SNP via bioinformatics tools (DIANA-microT v5.0 [159], PolymiRTS Database 3.0 [160], miRNASNP v2.0 [161], 
and RNAhybrid v2.1.2 software) to enlighten the molecular mechanisms that underlie the effect of DNMTs variants in gastric cancer. Yang et al. reported that DNMT3A rs13420827 is associated with gastric cancer risk [150], which was in disagreement with the results of two earlier studies $[137,153]$. Bioinformatics tools revealed that rs13420827 is potentially targeted by 3 different miRNAs which are depicted in figure 3 . The $\mathrm{C}$ allele generates putative miR-24$3 p$ and miR-4263 target sites while it disrupts miR-574-3p target site. MiR-574-3p participates in several cancers including colorectal, breast, lung, liver, and prostate cancer as a tumor suppressor. Su et al. used miRNA microarray and quantitative real-time PCR techniques, and found that miR-574-3p expression was down-regulated in gastric cancer patients at early stage or higher levels of differentiation. They also demonstrated that miR-574-3p suppresses cell proliferation, migration, and invasion of gastric cancer cells [162]. Recently, they also demonstrated that TGF- $\beta 1$-treated AGS cells show miR-574-3p up-regulation through binding of Mothers against decapentaplegic homolog 4 (SMAD4) to miR-574-3p promoter which might mediate the inhibition of cell proliferation in AGS cells by TGF- $\beta 1$ [163]. Moreover, mir-24-3p was demonstrated to be involved in gastric cancer inhibition and apoptosis induction, suggesting a tumor suppressive role for this miRNA. [164]. Thanks to the importance of SNPs located in crucial genes like DNMTs, further studies might validate their potential effect as a biomarker for early detection of gastric cancer.

\section{Dysregulation of $D N M T s$ in gastric cancer}

The 5-year relative survival rate for gastric cancer patients is $10-20 \%$, and the detection of the tumor at a late stage could be associated with poor prognosis and metastases in gastric cancer [165]. Mutation in DNMTs can cause some inherited diseases. DNMT1 mutations are associated with autosomal dominant cerebellar ataxia-deafness and narcolepsy (ADCA-DN), and hereditary sensory neuropathy with dementia and hearing loss [166]. ADCA-DN is a nervous system disorder with late onset (30-40 years old) caused by mutations in the C- 
terminus end of the DNMT1 gene. Mutations within the targeting-sequence domain of DNMT1 cause hereditary sensory neuropathy with dementia and hearing loss [167]. Generally, DNMT inhibition prevents DNA replication. Knock down of DNMT1 dysregulates P21 (a cyclin dependent kinase inhibitor 1, tumor suppressor) and the apoptosis inducer BIK (BCL2interacting killer) [168]. Another study shows that a cascade of genotoxic stress checkpoint proteins activates and induces cell cycle arrest following knock down of DNMT1 [168]. Mutations in DNMT3A may cause acute myeloid leukemia (AML) by epigenetic reactivation of the leukemogenic factor MEIS1 (myeloid ecotropic viral integration site 1 homolog) [169] Knockdown of DNMT3a was shown to inhibit embryonic cardiomyocytes function [170]. Also, depletion of DNMT3A may speed up lung tumor progression [171]. Aberrant promoter methylations found during early tumorigenesis are promising biomarkers for screening, early detection, and prognosis of cancer [172]. It has been indicated that hypermethylation of suppressor of cytokine signaling $1(S O C S-1)$ and death associated protein kinase 1 (DAPK) correlated with tumor stage [58, 173-174]. Also, Asada et al. used methylation level of miR124a-3, EMX1, and NKX6-1 to predict the risk of metachronous gastric cancer after endoscopic resection [175]. It seems that a better knowledge of genes involved in epigenetic alterations in gastric cancer can pave the way for designing an informative epigenetic biomarker panel for early detection of gastric cancer, and reduce mortality similar to what was accomplished in the bladder and lung cancers [176-177]. A series of methylated genes and correlation with clinical outcomes in gastric cancer are indicated in figure 4.

\section{DNMTs as target in gastric cancer chemotherapy}

One of the main treatment strategies for gastric cancer is chemotherapy. Commonly used chemo drugs for gastric cancer treatment include 5-FU (fluorouracil), cisplatin, irinotecan, and oxaliplatin [178]. The main problem with using this therapeutic approach is drug resistance. 
Drug resistance in cancer is related to various factors such as epigenetic changes, mutations, signaling pathways, and microenvironmental alterations. Methylation pattern alterations play important roles in response to treatment, and patient survival [179]. Many evidence showed the positive correlation between $D N M T$ s expression levels especially $D N M T 1$ and $D N M T 3 b$, with hypermethylation of $\mathrm{CpG}$ islands in gastric cancer [14]. Hypermethylated genes involved in chemotherapy resistance in gastric cancer are represented in figure 5. These genes are mainly involved in cell cycle regulation, genomic instability, epithelial-mesenchymal transition, apoptosis, and tumor suppression to eventually escape programmed cell death and acquire chemo-resistance (Fig 5).

Regarding the importance of DNA methylation in gastric cancer progression, anticancer drug sensitivity, and gastric cancer patient survival, DNMT inhibitors (DNMTis) can be useful in promoting the quality of treatment. Correspondingly, DNMT1 knockdown by short hairpin RNA in gastric cancer cell line led to increased chemo-sensitivity [180]. There are few studies on combining DNMTis with chemotherapy for gastric cancer [181-182]. However, the results are optimistic to accelerate remedy for this cancer by synergistic effect of DNMTis and anticancer drugs. Two clinical trials in phase I and phase II, focus on gastric cancer treatment with DNMTis [183-184]. In these clinical trials, researchers investigated the pretreatment with 5-azacytidine as hypomethylating agent in advanced gastrointestinal cancer [183]. They used 5-azacitidine (V) prior to EOX (epirubicin, oxaliplatin, capecitabine) neoadjuvant chemotherapy in gastric and esophageal adenocarcinoma. This study showed a hypomethylation of tumor-associated loci such as hyperpigmentation progressive 1 (HPP1), tissue inhibitor of metalloproteinases 3 (TIMP3), CDKN2A, estrogen receptor 1 (ESRl), and O-6-methylguanine-DNA methyltransferase $(M G M T)$. Neoadjuvant VEOX treatment was well-tolerated in all patients with significant clinical and epigenetic responses, with preliminary evidence that priming with $\mathrm{V}$ prior to chemotherapy may augment chemotherapy efficacy. 
DNA hypomethylation of tumor loci was seen at all dose levels, with greater staining for HPPI in the resected specimen compared with the pre-treatment specimen. Moreover, the expression of HPP1 as TSG marker was induced during neoadjuvant chemotherapy. 6 out of 12 treated patients became disease-free, had complete histologic response, and remained alive. [184]. DNMTis are divided into three general categories which include nucleoside analogs, nonnucleoside analogs, and nucleic acid-based components (Fig 6).

5-azacytidine and 5-aza-2'-deoxycytidine (decitabine) are chemical analogs of cytidine, and important DNMTi drugs approved by FDA for myelodysplastic syndrome (MDS), acute myeloid leukemia (AML), and chronic myelomonocytic leukemia (CMML) [185]. Many studies used azacytidine and decitabine as DNMTi for improving the treatment of gastric cancer alone or in combination with chemotherapy drugs such as 5-FU and cisplatin. Results of these studies demonstrate that azacytidine induces apoptosis and inhibits proliferation in gastric cancer cells, and improves the sensitivity of gastric cancer cells to 5-FU. Also, azacytidine upregulates death associated protein kinase 2 (DAPK2), DAPK3, RASSF1, and THBS1 genes that might be associated with the synergistic effect of chemotherapy. [186-188]. Other types of non-nucleoside analog or nucleic acid-based DNMTi's such as procainamide, procaine [189], hydrazone-gallate [190], genistein [191], microRNA-21 [192], microRNA-335 [193], microRNA-148a [192, 194], and microRNA-155-5p [195] were also used for targeting gastric cancer cells with or without anticancer drug, but further studies are necessary before approving their clinical use. Due to the side effect of chemical drugs, it has been demonstrated that DNMT-targeted inhibition with small interfering ribonucleic acids (siRNAs) may be used as a novel approach for reactivation of silenced genes. In support of this claim Jung et al. indicated that DNMT1 siRNAs inhibited cell proliferation and increased cell death rate in cancer cells without DNA damage, in comparison with azacytidine that increased DNA damage in human gastric adenocarcinoma cell lines $[50,196]$. Interestingly, all of these DNMTis 
enhanced the chemotherapy efficacy or programmed cell death in gastric cancer in in vitro and in vivo models. Table 3 summarizes DNMTis and their mechanism of action in gastric cancer.

Radiotherapy is another type of treatment which is commonly used for stage IV gastric cancer. In this method of treatment, the sensitivity and resistance of cells are important. Many studies showed the effects of epigenetic changes, especially methylation in cancer cells radioresistance [202]. Hypermethylation and inactivation of some genes involved in programmed cell death, cell cycle regulation, DNA repair, and TSGs can cause radiotherapy resistance in gastric cancer cells [188]. Treatment with 5-aza-2'-deoxycytidine (5-aza-CdR) showes a positive impact on gastric cancer cells radiosensitivity by increasing the expression of some genes such as $p 53$, $R A S S F 1$, and DAPK [203]. Nowadays, combination of routine cancer therapy methods such as radiotherapy or chemotherapy with epigenetic modulation drug is a hot topic in cancer therapy. The epigenetic modulation drugs had a powerful potential to improve cancer treatment. DNA methylation pattern changing can lead to increase or decrease of the expression of genes involved in tumorigenicity, invasion, and programmed cell death. Some clinical and preclinical data detected the toxicity of epigenetic modulating drugs, but other evidence indicate the benefits of epigenetic modulating drugs to cancer remedy. Therefore, in order to achieve an efficient radiotherapy or chemotherapy combination with epigenetic modulating drugs as well as DNMTis, a deeper knowledge of molecular mechanisms, long-term safety, effective dose of usage, limitations, off-targeting, and side effects of these drugs should be attained.

\section{Future Perspective}


Gastric cancer remains one of the major causes of morbidity and mortality in the world. Disruption of epigenetic processes can cause abnormal activation of oncogenes or silencing of TSGs as a hallmark of gastric cancer. Studies on epigenetic alterations occurring before or during gastric cancer have been the subject of great interest and could become a starting point for the development of future therapeutics tools in gastric cancer. Among epigenetic modifications, DNA methylation plays an important role in the tumorigenesis of gastric cancer. Emerging evidence shows that cancer cells exhibit aberrant DNA methylation relative to normal cells during gastric cancer development. The DNMTs are responsible for DNA methylation, and have a central role in epigenetic control of gene expression. Up-regulation of DNMTs leads to the increased hypermethylation of genes, which contributes to the promotion of tumor growth, invasion, and metastasis. Infections such as EBV, H. pylori, and HPV may directly effect tumorigenesis through secretion of proteins to increase the DNMTs expression in gastric cancer. Also, infections indirectly via TAMs increase DNMT1 expression. In addition to infections, some SNPs in DNMTs are associated with the survival rate of gastric cancer but little is known about miR-SNPs. Bioinformatics analysis revealed rs13420827 in the 3'-UTR of DNMT3A as part of a target site for three miRNAs including miR-24-3p, miR-4263, and miR-574-3p, which miR-24-3p and miR-574-3p have been found to be significantly associated with risk of gastric cancer. Moreover, epigenetic alterations are associated with chemoresistance in gastric cancer. Interestingly, as DNA methylation is a reversible process, restoration of the aberrant epigenetic changes may represent a promising strategy to overcoming chemo-resistance. Therefore, DNMTis such as shRNAs, miRNAs, 5-azacytidine, and 5-aza-2'-deoxycytidine can be useful in treating gastric cancer multidrug resistance. Another approach for improving efficacy is to combine the DNMTis with chemo drugs. However, epigenetic inhibitors such as 5-azacytidine and 5-aza-2'-deoxycytidine are not a specific inhibitor of DNMTs. CRISPR-Cas9 (clustered regularly interspaced short palindromic 
repeats-CRISPR associated nuclease 9) system has found a widespread use in biological and medical research, and becomes a promising strategy for editing DNA methylation. Recently, it has been indicated that CRISPR/Cas9 DNA methyltransferase fusion with a catalytically inactive Cas9 can lead to site-specific induction of DNA methylation [204-207]. Therefore, site-specific silencing of genes that contribute to the development of cancer via CRISPR/Cas9 system can be potentially superior to DNMTis, and appears to be a promising therapy in cancer patients.

\section{Executive Summary}

- DNA methyltransferases (DNMTs) consist of a catalytic and regulatory domain; the catalytic domain is conserved while the regulatory domain is variable.

- Aberrant expression of DNMTs is tightly associated with promoter hypermethylation of tumor suppressor genes.

- Infections such as EBV, HPV, and $H$. pylori facilitate DNMTs function by secreting oncogenic proteins or promoting differentiation and polarization of monocytes into tumorassociated macrophages.

- Genetic polymorphisms in DNMTs and their haplotype blocks may be potential biomarkers for gastric cancer prediction.

- Drug resistance in gastric cancer is triggered by hypermethylation of various genes.

- DNA hypomethylation of tumor loci via DNMT inhibitors may facilitate targeting gastric cancer multidrug resistance

- Site-specific DNA methylation through CRISPR/Cas9 system appears to be a promising targeting approach in cancerous cells.

\section{Conflict of interest}

Authors declare no conflict of interest. 


\section{References}

1. Sadikovic B, Al-Romaih K, Squire Ja, Zielenska M. Cause and consequences of genetic and epigenetic alterations in human cancer. Curr Genomics 9(6), 394-408 (2008).

2. Akhavan-Niaki H, Samadani Aa. DNA methylation and cancer development: molecular mechanism. Cell Biochem Biophys 67(2), 501-513 (2013).

3. Sharma S, Kelly Tk, Jones Pa. Epigenetics in cancer. Carcinogenesis 31(1), 27-36 (2010).

4. Resende C, Ristimaki A, Machado Jc. Genetic and epigenetic alteration in gastric carcinogenesis. Helicobacter 15 Suppl 1 34-39 (2010).

5. Nardone G, Compare D. Epigenetic alterations due to diet and Helicobacter pylori infection in gastric carcinogenesis. Expert Review of Gastroenterology \& Hepatology 2(2), 243-248 (2008).

6. Yang Wy, Gu Jl, Zhen Tm. Recent advances of histone modification in gastric cancer. J Cancer Res Ther 10 Suppl 240-245 (2014).

7. Zhang M, Du X. Noncoding RNAs in gastric cancer: Research progress and prospects. World J Gastroenterol 22(29), 6610-6618 (2016).

8. Tahara T, Arisawa T. DNA methylation as a molecular biomarker in gastric cancer. Epigenomics 7(3), 475-486 (2015).

9. Kunej T, Godnic I, Ferdin J, Horvat S, Dovc P, Calin Ga. Epigenetic regulation of microRNAs in cancer: an integrated review of literature. Mutat Res 717(1-2), 77-84 (2011).

10. Wajed Sa, Laird Pw, Demeester Tr. DNA methylation: an alternative pathway to cancer. Ann Surg 234(1), 10-20 (2001).

11. Fernandez Af, Huidobro C, Fraga Mf. De novo DNA methyltransferases: oncogenes, tumor suppressors, or both? Trends Genet 28(10), 474-479 (2012).

12. Fu Dg. Epigenetic alterations in gastric cancer (Review). Mol Med Rep 12(3), 3223-3230 (2015).

13. Qu Y, Dang S, Hou P. Gene methylation in gastric cancer. Clinica Chimica Acta 424 53-65 (2013). 
14. Yang J, Wei X, Wu Q et al. Clinical significance of the expression of DNA methyltransferase proteins in gastric cancer. Mol Med Rep 4(6), 1139-1143 (2011).

15. Ding Wj, Fang Jy, Chen Xy, Peng Ys. The expression and clinical significance of DNA methyltransferase proteins in human gastric cancer. Dig Dis Sci 53(8), 2083-2089 (2008).

16. Luczak Mw, Jagodzinski Pp. The role of DNA methylation in cancer development. Folia Histochem Cytobiol 44(3), 143-154 (2006).

17. Mccabe Mt, Brandes Jc, Vertino Pm. Cancer DNA methylation: molecular mechanisms and clinical implications. Clin Cancer Res 15(12), 3927-3937 (2009).

18. Lyko F. The DNA methyltransferase family: a versatile toolkit for epigenetic regulation. Nat Rev Genet 19(2), 81-92 (2018).

** A very recent article that describes the structure of different DNMTs in mammals, their function, and different mechanisms regulating their activity.

19. Rountree Mr, Bachman Ke, Baylin Sb. DNMT1 binds HDAC2 and a new co-repressor, DMAP1, to form a complex at replication foci. Nat Genet 25(3), 269-277 (2000).

20. Egger G, Jeong S, Escobar Sg et al. Identification of DNMT1 (DNA methyltransferase 1) hypomorphs in somatic knockouts suggests an essential role for DNMT1 in cell survival. Proc Natl Acad Sci U S A 103(38), 14080-14085 (2006).

21. Garvilles Rg, Hasegawa T, Kimura H et al. Dual Functions of the RFTS Domain of Dnmt1 in Replication-Coupled DNA Methylation and in Protection of the Genome from Aberrant Methylation. PloS one 10(9), e0137509 (2015).

22. Syeda F, Fagan Rl, Wean M et al. The replication focus targeting sequence (RFTS) domain is a DNA-competitive inhibitor of Dnmt1. J Biol Chem 286(17), 15344-15351 (2011).

23. Pradhan M, Esteve Po, Chin Hg, Samaranayke M, Kim Gd, Pradhan S. CXXC domain of human DNMT1 is essential for enzymatic activity. Biochemistry 47(38), 10000-10009 (2008).

24. Callebaut I, Courvalin Jc, Mornon Jp. The BAH (bromo-adjacent homology) domain: a link between DNA methylation, replication and transcriptional regulation. FEBS Lett 446(1), 189193 (1999). 
25. Jeltsch A, Jurkowska Rz. Allosteric control of mammalian DNA methyltransferases - a new regulatory paradigm. Nucleic Acids Res 44(18), 8556-8575 (2016).

* Describes the structure, mechanisms of function and regulation of the activity of DNMT1 and DNMT3.

26. Okano M, Xie S, Li E. Cloning and characterization of a family of novel mammalian DNA (cytosine-5) methyltransferases. Nat Genet 19(3), 219-220 (1998).

27. Xie S, Wang Z, Okano M et al. Cloning, expression and chromosome locations of the human DNMT3 gene family. Gene 236(1), 87-95 (1999).

28. Dhayalan A, Rajavelu A, Rathert P et al. The Dnmt3a PWWP domain reads histone 3 lysine 36 trimethylation and guides DNA methylation. J Biol Chem 285(34), 26114-26120 (2010).

29. Chen T, Tsujimoto N, Li E. The PWWP domain of Dnmt3a and Dnmt3b is required for directing DNA methylation to the major satellite repeats at pericentric heterochromatin. $\mathrm{Mol}$ Cell Biol 24(20), 9048-9058 (2004).

30. Argentaro A, Yang Jc, Chapman L et al. Structural consequences of disease-causing mutations in the ATRX-DNMT3-DNMT3L (ADD) domain of the chromatin-associated protein ATRX. Proc Natl Acad Sci U S A 104(29), 11939-11944 (2007).

31. Dhayalan A, Tamas R, Bock I et al. The ATRX-ADD domain binds to H3 tail peptides and reads the combined methylation state of K4 and K9. Hum Mol Genet 20(11), 2195-2203 (2011).

32. Otani J, Nankumo T, Arita K, Inamoto S, Ariyoshi M, Shirakawa M. Structural basis for recognition of H3K4 methylation status by the DNA methyltransferase 3A ATRX-DNMT3DNMT3L domain. EMBO Rep 10(11), 1235-1241 (2009).

33. Toyota M, Yamamoto E. DNA methylation changes in cancer. Prog Mol Biol Transl Sci 101 447-457 (2011).

34. Wang H, Duan Xl, Qi Xl et al. Concurrent Hypermethylation of SFRP2 and DKK2 Activates the Wnt/beta-Catenin Pathway and Is Associated with Poor Prognosis in Patients with Gastric Cancer. Mol Cells 40(1), 45-53 (2017). 
35. Chiurillo Ma. Role of the Wnt/beta-catenin pathway in gastric cancer: An in-depth literature review. World J Exp Med 5(2), 84-102 (2015).

36. Ersahin T, Tuncbag N, Cetin-Atalay R. The PI3K/AKT/mTOR interactive pathway. Mol Biosyst 11(7), 1946-1954 (2015).

37. Matsuoka T, Yashiro M. The Role of PI3K/Akt/mTOR Signaling in Gastric Carcinoma. Cancers (Basel) 6(3), 1441-1463 (2014).

38. Du W, Wang S, Zhou Q et al. ADAMTS9 is a functional tumor suppressor through inhibiting AKT/mTOR pathway and associated with poor survival in gastric cancer. Oncogene 32(28), 3319-3328 (2013).

39. Singh Ss, Yap Wn, Arfuso F et al. Targeting the PI3K/Akt signaling pathway in gastric carcinoma: A reality for personalized medicine? World J Gastroenterol 21(43), 12261-12273 (2015).

40. Huang T, Zhou Y, Cheng As, Yu J, To Kf, Kang W. NOTCH receptors in gastric and other gastrointestinal cancers: oncogenes or tumor suppressors? Mol Cancer 15(1), 80 (2016).

41. Piazzi G, Fini L, Selgrad M et al. Epigenetic regulation of Delta-Like1 controls Notch1 activation in gastric cancer. Oncotarget 2(12), 1291-1301 (2011).

42. Piazzi G, Bazzoli F, Ricciardiello L. Epigenetic silencing of Notch signaling in gastrointestinal cancers. Cell Cycle 11(23), 4323-4327 (2012).

43. Yeh Ts, $\mathrm{Wu} \mathrm{Cw}, \mathrm{Hsu} \mathrm{Kw}$ et al. The activated Notch1 signal pathway is associated with gastric cancer progression through cyclooxygenase-2. Cancer Res 69(12), 5039-5048 (2009).

44. Brzozowa M, Mielanczyk L, Michalski M et al. Role of Notch signaling pathway in gastric cancer pathogenesis. Contemp Oncol (Pozn) 17(1), 1-5 (2013).

45. Yao Y, Ni Y, Zhang J, Wang H, Shao S. The role of Notch signaling in gastric carcinoma: molecular pathogenesis and novel therapeutic targets. Oncotarget 8(32), 53839-53853 (2017).

46. Yu W, Li X, Eliason S et al. Irx1 regulates dental outer enamel epithelial and lung alveolar type II epithelial differentiation. Dev Biol 429(1), 44-55 (2017).

47. Guo X, Liu W, Pan Y et al. Homeobox gene IRX1 is a tumor suppressor gene in gastric carcinoma. Oncogene 29(27), 3908-3920 (2010). 
48. Lai J, Wang H, Luo Q et al. The relationship between DNA methylation and Reprimo gene expression in gastric cancer cells. Oncotarget 8(65), 108610-108623 (2017).

49. Etoh T, Kanai Y, Ushijima $\mathrm{S}$ et al. Increased DNA methyltransferase 1 (DNMT1) protein expression correlates significantly with poorer tumor differentiation and frequent DNA hypermethylation of multiple CpG islands in gastric cancers. Am J Pathol 164(2), 689-699 (2004).

50. Jung Y, Park J, Kim Ty et al. Potential advantages of DNA methyltransferase 1 (DNMT1)targeted inhibition for cancer therapy. J Mol Med (Berl) 85(10), 1137-1148 (2007).

51. Waki T, Tamura G, Tsuchiya T, Sato K, Nishizuka S, Motoyama T. Promoter methylation status of E-cadherin, hMLH1, and p16 genes in nonneoplastic gastric epithelia. Am J Pathol 161(2), 399-403 (2002).

52. Deng J, Liang H, Zhang R et al. Applicability of the methylated CpG sites of paired box 5 (PAX5) promoter for prediction the prognosis of gastric cancer. Oncotarget 5(17), 7420-7430 (2014).

53. Cheng As, Li Ms, Kang W et al. Helicobacter pylori causes epigenetic dysregulation of FOXD3 to promote gastric carcinogenesis. Gastroenterology 144(1), 122-133 e129 (2013).

54. Shi H, Wang X, Wang J, Pan J, Liu J, Ye B. Association between CHFR gene hypermethylation and gastric cancer risk: a meta-analysis. Onco Targets Ther 9 7409-7414 (2016).

55. Chen Hy, Zhu Bh, Zhang Ch et al. High CpG island methylator phenotype is associated with lymph node metastasis and prognosis in gastric cancer. Cancer Sci 103(1), 73-79 (2012).

56. Yamashita S, Tsujino Y, Moriguchi K, Tatematsu M, Ushijima T. Chemical genomic screening for methylation-silenced genes in gastric cancer cell lines using 5-aza-2'-deoxycytidine treatment and oligonucleotide microarray. Cancer Sci 97(1), 64-71 (2006).

57. Li Y, Yang Y, Lu Y et al. Predictive value of CHFR and MLH1 methylation in human gastric cancer. Gastric Cancer 18(2), 280-287 (2015).

58. Jia W, Yu T, Cao X, An Q, Yang H. Clinical effect of DAPK promoter methylation in gastric cancer: A systematic meta-analysis. Medicine (Baltimore) 95(43), e5040 (2016). 
59. Zazula M, Ferreira Am, Czopek Jp et al. CDH1 gene promoter hypermethylation in gastric cancer: relationship to Goseki grading, microsatellite instability status, and EBV invasion. Diagn Mol Pathol 15(1), 24-29 (2006).

60. Tao K, Wu C, Wu K et al. Quantitative analysis of promoter methylation of the EDNRB gene in gastric cancer. Med Oncol 29(1), 107-112 (2012).

61. Buffart Te, Overmeer Rm, Steenbergen Rd et al. MAL promoter hypermethylation as a novel prognostic marker in gastric cancer. Br J Cancer 99(11), 1802-1807 (2008).

62. Hu Xt, Zhang Fb, Fan Yc et al. Phospholipase $\mathrm{C}$ delta 1 is a novel 3p22.3 tumor suppressor involved in cytoskeleton organization, with its epigenetic silencing correlated with high-stage gastric cancer. Oncogene 28(26), 2466-2475 (2009).

63. Kim M, Jang Hr, Haam K et al. Frequent silencing of popeye domain-containing genes, BVES and POPDC3, is associated with promoter hypermethylation in gastric cancer. Carcinogenesis 31(9), 1685-1693 (2010).

64. Li Z, Chim Jc, Yang M, Ye J, Wong Bc, Qiao L. Role of PCDH10 and its hypermethylation in human gastric cancer. Biochim Biophys Acta 1823(2), 298-305 (2012).

65. Oishi Y, Watanabe Y, Yoshida Y et al. Hypermethylation of Sox17 gene is useful as a molecular diagnostic application in early gastric cancer. Tumour Biol 33(2), 383-393 (2012).

66. Liu X, Wang X, Zhang J et al. Warburg effect revisited: an epigenetic link between glycolysis and gastric carcinogenesis. Oncogene 29(3), 442-450 (2010).

67. Kim M, Kim Jh, Jang $\mathrm{Hr}$ et al. LRRC3B, encoding a leucine-rich repeat-containing protein, is a putative tumor suppressor gene in gastric cancer. Cancer Res 68(17), 7147-7155 (2008).

68. Cheng Yy, Jin H, Liu X et al. Fibulin 1 is downregulated through promoter hypermethylation in gastric cancer. Br J Cancer 99(12), 2083-2087 (2008).

69. Wanajo A, Sasaki A, Nagasaki H et al. Methylation of the calcium channel-related gene, CACNA2D3, is frequent and a poor prognostic factor in gastric cancer. Gastroenterology 135(2), 580-590 (2008). 
70. Wu Cs, $\mathrm{Lu} \mathrm{Yj,} \mathrm{Li} \mathrm{Hp} \mathrm{et} \mathrm{al.} \mathrm{Glutamate} \mathrm{receptor,} \mathrm{ionotropic,} \mathrm{kainate} 2$ silencing by DNA hypermethylation possesses tumor suppressor function in gastric cancer. Int J Cancer 126(11), 2542-2552 (2010).

71. Dong W, Feng L, Xie Y, Zhang H, Wu Y. Hypermethylation-mediated reduction of LMX1A expression in gastric cancer. Cancer Sci 102(2), 361-366 (2011).

72. Chen H, Pan Y, Cheng Zy et al. Hypermethylation and clinicopathological significance of RASAL1 gene in gastric cancer. Asian Pac J Cancer Prev 14(11), 6261-6265 (2013).

73. Jiang P, Yu G, Zhang Y et al. Promoter hypermethylation and downregulation of trefoil factor 2 in human gastric cancer. Oncol Lett 7(5), 1525-1531 (2014).

74. Waraya M, Yamashita $\mathrm{K}$, Katoh $\mathrm{H}$ et al. Cancer specific promoter $\mathrm{CpG}$ Islands hypermethylation of HOP homeobox (HOPX) gene and its potential tumor suppressive role in pancreatic carcinogenesis. BMC Cancer 12397 (2012).

75. Akiyama Y, Watkins N, Suzuki H et al. GATA-4 and GATA-5 transcription factor genes and potential downstream antitumor target genes are epigenetically silenced in colorectal and gastric cancer. Mol Cell Biol 23(23), 8429-8439 (2003).

76. Du Yy, Dai Dq, Yang Z. Role of RECK methylation in gastric cancer and its clinical significance. World J Gastroenterol 16(7), 904-908 (2010).

77. Kim H, Kim Yh, Kim Se, Kim Ng, Noh Sh, Kim H. Concerted promoter hypermethylation of hMLH1, p16INK4A, and E-cadherin in gastric carcinomas with microsatellite instability. $J$ Pathol 200(1), 23-31 (2003).

78. Dohi O, Takada H, Wakabayashi $\mathrm{N}$ et al. Epigenetic silencing of RELN in gastric cancer. Int $J$ Oncol 36(1), 85-92 (2010).

79. Du P, Ye Hr, Gao J et al. Methylation of PTCH1a gene in a subset of gastric cancers. World J Gastroenterol 15(30), 3799-3806 (2009).

80. Kim M, Lee Kt, Jang Hr et al. Epigenetic down-regulation and suppressive role of DCBLD2 in gastric cancer cell proliferation and invasion. Mol Cancer Res 6(2), 222-230 (2008).

81. Wang G, Zhang W, Zhou B et al. The diagnosis value of promoter methylation of UCHL1 in the serum for progression of gastric cancer. Biomed Res Int 2015741030 (2015). 
82. Mikata R, Fukai K, Imazeki $\mathrm{F}$ et al. BCL2L10 is frequently silenced by promoter hypermethylation in gastric cancer. Oncol Rep 23(6), 1701-1708 (2010).

83. Shi X, Li X, Chen L, Wang C. Analysis of somatostatin receptors and somatostatin promoter methylation in human gastric cancer. Oncol Lett 6(6), 1794-1798 (2013).

84. Liu Y, Chew Mh, Tham Ck, Tang Cl, Ong Sy, Zhao Y. Methylation of serum SST gene is an independent prognostic marker in colorectal cancer. Am J Cancer Res 6(9), 2098-2108 (2016).

85. Wen Xz, Akiyama Y, Baylin Sb, Yuasa Y. Frequent epigenetic silencing of the bone morphogenetic protein 2 gene through methylation in gastric carcinomas. Oncogene 25(18), 2666-2673 (2006).

86. Kim Sk, Jang Hr, Kim Jh et al. The epigenetic silencing of LIMS2 in gastric cancer and its inhibitory effect on cell migration. Biochem Biophys Res Commun 349(3), 1032-1040 (2006).

87. Kim St, Jang Hl, Lee J et al. Clinical Significance of IGFBP-3 Methylation in Patients with Early Stage Gastric Cancer. Transl Oncol 8(4), 288-294 (2015).

88. Wang Lh, Kim Sh, Lee Jh et al. Inactivation of SMAD4 tumor suppressor gene during gastric carcinoma progression. Clin Cancer Res 13(1), 102-110 (2007).

89. Noda H, Miyaji Y, Nakanishi A, Konishi F, Miki Y. Frequent reduced expression of alpha-1Badrenergic receptor caused by aberrant promoter methylation in gastric cancers. $\mathrm{Br} \mathrm{J}$ Cancer 96(2), 383-390 (2007).

90. Kato K, lida S, Uetake H et al. Methylated TMS1 and DAPK genes predict prognosis and response to chemotherapy in gastric cancer. Int J Cancer 122(3), 603-608 (2008).

91. Su Y, Lin Y, Zhang L et al. CMTM3 inhibits cell migration and invasion and correlates with favorable prognosis in gastric cancer. Cancer Sci 105(1), 26-34 (2014).

92. Kim J, Min Sy, Lee He, Kim Wh. Aberrant DNA methylation and tumor suppressive activity of the EBF3 gene in gastric carcinoma. Int J Cancer 130(4), 817-826 (2012).

93. Leung $\mathrm{Wk}$, Yu J, Bai Ah et al. Inactivation of helicase-like transcription factor by promoter hypermethylation in human gastric cancer. Mol Carcinog 37(2), 91-97 (2003).

94. Akino K, Toyota M, Suzuki $\mathrm{H}$ et al. Identification of DFNA5 as a target of epigenetic inactivation in gastric cancer. Cancer Sci 98(1), 88-95 (2007). 
95. Da Mx, Zhang Yb, Yao Jb, Duan Yx. DNA methylation regulates expression of VEGF-C, and S-adenosylmethionine is effective for VEGF-C methylation and for inhibiting cancer growth. Braz J Med Biol Res 47(12), 1021-1028 (2014).

96. Jin Sh, Akiyama Y, Fukamachi H, Yanagihara K, Akashi T, Yuasa Y. IQGAP2 inactivation through aberrant promoter methylation and promotion of invasion in gastric cancer cells. Int $J$ Cancer 122(5), 1040-1046 (2008).

97. Otsubo T, Akiyama Y, Yanagihara K, Yuasa Y. SOX2 is frequently downregulated in gastric cancers and inhibits cell growth through cell-cycle arrest and apoptosis. Br J Cancer 98(4), 824-831 (2008).

98. Wang L, Zhu Js, Song Mq, Chen Gq, Chen Jl. Comparison of gene expression profiles between primary tumor and metastatic lesions in gastric cancer patients using laser microdissection and cDNA microarray. World J Gastroenterol 12(43), 6949-6954 (2006).

99. Cui Y, Ying Y, Van Hasselt A et al. OPCML is a broad tumor suppressor for multiple carcinomas and lymphomas with frequently epigenetic inactivation. PLoS One 3(8), e2990 (2008).

100. Fang Dc, Luo Yh, Yang Sm, Li Xa, Ling Xl, Fang L. Mutation analysis of APC gene in gastric cancer with microsatellite instability. World J Gastroenterol 8(5), 787-791 (2002).

101. Campbell Pm, Szyf M. Human DNA methyltransferase gene DNMT1 is regulated by the APC pathway. Carcinogenesis 24(1), 17-24 (2003).

102. Hmadcha A, Bedoya Fj, Sobrino F, Pintado E. Methylation-dependent gene silencing induced by interleukin 1 beta via nitric oxide production. J Exp Med 190(11), 1595-1604 (1999).

103. Huang Fy, Chan Ao, Rashid A, Wong Dk, Cho Ch, Yuen Mf. Helicobacter pylori induces promoter methylation of E-cadherin via interleukin-1beta activation of nitric oxide production in gastric cancer cells. Cancer 118(20), 4969-4980 (2012).

104. Fattahi S, Nikbakhsh N, Taheri H et al. Prevalence of multiple infections and the risk of gastric adenocarcinoma development at earlier age. Diagn Microbiol Infect Dis (2018).

105. Fattahi S, Kosari-Monfared M, Ghadami E et al. Infection-associated epigenetic alterations in gastric cancer: New insight in cancer therapy. J Cell Physiol (2018). 
106. Zeng Zm, Luo Ff, Zou Lx et al. Human papillomavirus as a potential risk factor for gastric cancer: a meta-analysis of 1,917 cases. Onco Targets Ther 9 7105-7114 (2016).

107. Snietura M, Waniczek D, Piglowski W et al. Potential role of human papilloma virus in the pathogenesis of gastric cancer. World J Gastroenterol 20(21), 6632-6637 (2014).

108. Au Yeung Cl, Tsang Wp, Tsang Ty, Co Nn, Yau Pl, Kwok Tt. HPV-16 E6 upregulation of DNMT1 through repression of tumor suppressor p53. Oncology reports 24(6), 1599-1604 (2010).

109. Lin $\mathrm{Rk}$, Wu Cy, Chang $\mathrm{Jw}$ et al. Dysregulation of $\mathrm{p} 53 / \mathrm{Sp} 1$ control leads to DNA methyltransferase-1 overexpression in lung cancer. Cancer Res 70(14), 5807-5817 (2010).

110. Tang Y-A, Tsai Y-T, Lin R-K, Hsu H-S, Chen C-Y, Wang Y-C. Deregulation of p53 and RB Transcriptional Control Leads to Overexpression of DNA Methyltransferases in Lung Cancer. Journal of Cancer Research and Practice 1(1), 14-27 (2014).

111. Zhang Bg, Hu L, Zang Md et al. Helicobacter pylori CagA induces tumor suppressor gene hypermethylation by upregulating DNMT1 via AKT-NFkappaB pathway in gastric cancer development. Oncotarget 7(9), 9788-9800 (2016).

112. Wang H-C, Chen C-W, Yang C-L et al. Tumor-Associated Macrophages Promote Epigenetic Silencing of Gelsolin through DNA Methyltransferase 1 in Gastric Cancer Cells. Cancer Immunology Research 5(10), 885-897 (2017).

* Demonstrates the impact of chronic inflammation on gastric cancer progression via aberrant DNA methylation of the gelsolin gene.

113. Baj-Krzyworzeka M, Weglarczyk K, Baran J, Szczepanik A, Szura M, Siedlar M. Elevated level of some chemokines in plasma of gastric cancer patients. Cent Eur J Immunol 41(4), 358362 (2016).

114. Wang T, Wei Y, Tian L et al. C-C motif chemokine ligand 5 (CCL5) levels in gastric cancer patient sera predict occult peritoneal metastasis and a poorer prognosis. Int J Surg 32 136-142 (2016). 
115. Kudo T, Lu H, Wu Jy, Graham Dy, Casola A, Yamaoka Y. Regulation of RANTES promoter activation in gastric epithelial cells infected with Helicobacter pylori. Infect Immun 73(11), 7602-7612 (2005).

116. Nakayama T, Hieshima K, Nagakubo D et al. Selective induction of Th2-attracting chemokines CCL17 and CCL22 in human B cells by latent membrane protein 1 of Epstein-Barr virus. $J$ Virol 78(4), 1665-1674 (2004).

117. Westrich Ja, Warren Cj, Pyeon D. Evasion of host immune defenses by human papillomavirus. Virus Research 231 21-33 (2017).

118. Cao Xy, Ma Hx, Shang Yh et al. DNA methyltransferase3a expression is an independent poor prognostic indicator in gastric cancer. World J Gastroenterol 20(25), 8201-8208 (2014).

119. Huang F-Y, Chan Ao-O, Lo Rc-L et al. Characterization of interleukin-1 $\beta$ in Helicobacter pylori-induced gastric inflammation and DNA methylation in interleukin-1 receptor type 1 knockout (IL-1R1-/-) mice. European Journal of Cancer 49(12), 2760-2770 (2013).

120. El-Omar Em, Carrington M, Chow Wh et al. Interleukin-1 polymorphisms associated with increased risk of gastric cancer. Nature 404(6776), 398-402 (2000).

121. Valenzuela Ma, Canales J, Corvalan Ah, Quest Af. Helicobacter pylori-induced inflammation and epigenetic changes during gastric carcinogenesis. World J Gastroenterol 21(45), 1274212756 (2015).

122. Ishii Y, Shibata W, Sugimori M et al. Activation of Signal Transduction and Activator of Transcription 3 Signaling Contributes to Helicobacter-Associated Gastric Epithelial Proliferation and Inflammation. Gastroenterol Res Pract 20189050715 (2018).

123. Kang Hj, Yi Yw, Hou Sj et al. Disruption of STAT3-DNMT1 interaction by SH-I-14 induces re-expression of tumor suppressor genes and inhibits growth of triple-negative breast tumor. Oncotarget 8(48), 83457-83468 (2017).

124. Maekita T, Nakazawa K, Mihara M et al. High levels of aberrant DNA methylation in Helicobacter pylori-infected gastric mucosae and its possible association with gastric cancer risk. Clin Cancer Res 12(3 Pt 1), 989-995 (2006). 
125. Ando T, Yoshida T, Enomoto S et al. DNA methylation of microRNA genes in gastric mucosae of gastric cancer patients: its possible involvement in the formation of epigenetic field defect. Int J Cancer 124(10), 2367-2374 (2009).

126. Niwa $\mathrm{T}$, Tsukamoto $\mathrm{T}$, Toyoda $\mathrm{T}$ et al. Inflammatory processes triggered by Helicobacter pylori infection cause aberrant DNA methylation in gastric epithelial cells. Cancer Res 70(4), 1430$1440(2010)$.

127. Maeda M, Moro H, Ushijima T. Mechanisms for the induction of gastric cancer by Helicobacter pylori infection: aberrant DNA methylation pathway. Gastric Cancer 20(Suppl 1), 8-15 (2017).

128. Piao Jy, Lee Hg, Kim Sj et al. Helicobacter pylori Activates IL-6-STAT3 Signaling in Human Gastric Cancer Cells: Potential Roles for Reactive Oxygen Species. Helicobacter 21(5), 405416 (2016).

129. Zhang Q, Wang Hy, Woetmann A, Raghunath Pn, Odum N, Wasik Ma. STAT3 induces transcription of the DNA methyltransferase 1 gene (DNMT1) in malignant T lymphocytes. Blood 108(3), 1058-1064 (2006).

130. Lee H, Zhang P, Herrmann A et al. Acetylated STAT3 is crucial for methylation of tumorsuppressor gene promoters and inhibition by resveratrol results in demethylation. Proc Natl Acad Sci U S A 109(20), 7765-7769 (2012).

131. Wang $\mathrm{Hc}$, Chen $\mathrm{Cw}$, Yang $\mathrm{Cl}$ et al. Tumor-Associated Macrophages Promote Epigenetic Silencing of Gelsolin through DNA Methyltransferase 1 in Gastric Cancer Cells. Cancer Immunol Res 5(10), 885-897 (2017).

132. Hino R, Uozaki H, Murakami $\mathrm{N}$ et al. Activation of DNA methyltransferase 1 by EBV latent membrane protein 2A leads to promoter hypermethylation of PTEN gene in gastric carcinoma. Cancer Res 69(7), 2766-2774 (2009).

133. Zhao J, Liang Q, Cheung Kf et al. Genome-wide identification of Epstein-Barr virus-driven promoter methylation profiles of human genes in gastric cancer cells. Cancer 119(2), 304-312 (2013).

134. Tsai Cl, Li Hp, Lu Yj et al. Activation of DNA methyltransferase 1 by EBV LMP1 Involves cJun NH(2)-terminal kinase signaling. Cancer Res 66(24), 11668-11676 (2006). 
135. Li H, Li W, Liu S et al. DNMT1, DNMT3A and DNMT3B Polymorphisms Associated With Gastric Cancer Risk: A Systematic Review and Meta-analysis. EBioMedicine 13 125-131 (2016).

136. Shastry Bs. SNPs: impact on gene function and phenotype. Methods in molecular biology (Clifton, N.J.) 578 3-22 (2009).

137. Wang C, Jia Z, Ma H et al. DNA methyltransferase 3a rs1550117 genetic polymorphism predicts poor survival in gastric cancer patients. International journal of clinical and experimental pathology 8(11), 14864-14874 (2015).

138. Zhang Y, Xu H, Shen Y, Gong Z, Xiao T. Association of DNMT3B -283 T > C and -579 G > $\mathrm{T}$ polymorphisms with decreased cancer risk: evidence from a meta-analysis. Int J Clin Exp Med 8(8), 13028-13038 (2015).

139. Zhu J, Du S, Zhang J, Wang Y, Wu Q, Ni J. Polymorphism of DNA methyltransferase 3B 149C/T and cancer risk: a meta-analysis. Med Oncol 32(1), 399 (2015).

140. Liu Z, Wang L, Wang Le, Sturgis Em, Wei Q. Polymorphisms of the DNMT3B gene and risk of squamous cell carcinoma of the head and neck: a case-control study. Cancer letters 268(1), $158-165$ (2008).

141. Singal R, Das Pm, Manoharan M, Reis Im, Schlesselman Jj. Polymorphisms in the DNA methyltransferase 3b gene and prostate cancer risk. Oncology reports 14(2), 569-573 (2005).

142. Montgomery Kg, Liu Mc, Eccles Dm, Campbell Ig. The DNMT3B C-->T promoter polymorphism and risk of breast cancer in a British population: a case-control study. Breast cancer research : BCR 6(4), R390-394 (2004).

143. Zhang S-H, Yang L-H, Ma X-M. Association between SNP (149C/T) of DNMT3B gene and the risk of lung cancer in Jiamusi. Chin J Gerontol 31 4090-4092 (2011).

144. Hu J, Fan H, Liu D, Zhang S, Zhang F, Xu H. DNMT3B promoter polymorphism and risk of gastric cancer. Digestive diseases and sciences 55(4), 1011-1016 (2010).

145. Chen B, Wang J, Gu X, Zhang J, Zhang J, Feng X. The DNMT3B -579G>T Polymorphism Is Significantly Associated With the Risk of Gastric Cancer but not Lung Cancer in Chinese Population. Technology in cancer research \& treatment 16(6), 1259-1265 (2017). 
146. Zhou J, Zhi X, Wang L et al. Linc00152 promotes proliferation in gastric cancer through the EGFR-dependent pathway. Journal of experimental \& clinical cancer research : CR 34135 (2015).

147. Ahmadi K, Soleimani A, Irani S et al. DNMT3B -579 G>T Promoter Polymorphism and the Risk of Gastric Cancer in the West of Iran. Journal of gastrointestinal cancer (2017).

148. Aung Pp, Matsumura S, Kuraoka K et al. No evidence of correlation between the single nucleotide polymorphism of DNMT3B promoter and gastric cancer risk in a Japanese population. Oncology reports 14(5), 1151-1154 (2005).

149. Wang Ym, Wang R, Wen Dg et al. Single nucleotide polymorphism in DNA methyltransferase 3B promoter and its association with gastric cardiac adenocarcinoma in North China. World $J$ Gastroenterol 11(23), 3623-3627 (2005).

150. Yang Xx, He Xq, Li Fx, Wu Ys, Gao Y, Li M. Risk-association of DNA methyltransferases polymorphisms with gastric cancer in the Southern Chinese population. International journal of molecular sciences 13(7), 8364-8378 (2012).

151. Jia Z, Wu X, Cao D et al. Polymorphisms of the DNA Methyltransferase 1 Gene Predict Survival of Gastric Cancer Patients Receiving Tumorectomy. Disease markers 20168578064 (2016).

152. Jiang J, Jia Z, Cao D et al. Polymorphisms of the DNA methyltransferase 1 associated with reduced risks of Helicobacter pylori infection and increased risks of gastric atrophy. PloS one 7(9), e46058 (2012).

153. Cao Xy, Jia Zf, Cao Dh et al. DNMT3a rs1550117 polymorphism association with increased risk of Helicobacter pylori infection. Asian Pacific journal of cancer prevention : APJCP 14(10), 5713-5718 (2013).

154. Wu H, Zhang K, Gong P et al. A novel functional TagSNP Rs7560488 in the DNMT3A1 promoter is associated with susceptibility to gastric cancer by modulating promoter activity. PloS one 9(3), e92911 (2014).

155. Wu Q, Lu S, Wang L et al. DNMT3A rs36012910 A>G polymorphism and gastric cancer susceptibility in a Chinese population. Molecular biology reports 39(12), 10949-10955 (2012). 
156. Fan H, Liu D, Qiu X et al. A functional polymorphism in the DNA methyltransferase-3A promoter modifies the susceptibility in gastric cancer but not in esophageal carcinoma. $B M C$ medicine 812 (2010).

157. Khatami F, Noorinayer B, Ghiasi S, Mohebi R, Hashemi M, Zali Mr. Lack of effects of single nucleotide polymorphisms of the DNA methyltransferase 1 gene on gastric cancer in Iranian patients: a case control study. Asian Pacific journal of cancer prevention : APJCP 10(6), 1177$1182(2009)$.

158. Wang C, Jia Z, Cao D et al. Polymorphism of DNA Methyltransferase $3 \mathrm{~b}$ and Association with Development and Prognosis in Gastric Cancer. PloS one 10(8), e0134059 (2015).

159. Paraskevopoulou Md, Georgakilas G, Kostoulas N et al. DIANA-microT web server v5. 0: service integration into miRNA functional analysis workflows. Nucleic acids research 41(W1), W169-W173 (2013).

160. Bhattacharya A, Ziebarth Jd, Cui Y. PolymiRTS Database 3.0: linking polymorphisms in microRNAs and their target sites with human diseases and biological pathways. Nucleic acids research 42(D1), D86-D91 (2013).

161. Gong J, Liu C, Liu W et al. An update of miRNASNP database for better SNP selection by GWAS data, miRNA expression and online tools. Database 2015 (2015).

162. Su Y, Ni Z, Wang G et al. Aberrant expression of microRNAs in gastric cancer and biological significance of miR-574-3p. International immunopharmacology 13(4), 468-475 (2012).

163. Zhang R, Wang M, Sui P, Ding L, Yang Q. Upregulation of microRNA-574-3p in a human gastric cancer cell line AGS by TGF-beta1. Gene 605 63-69 (2017).

164. Duan Y, Hu L, Liu B et al. Tumor suppressor miR-24 restrains gastric cancer progression by downregulating RegIV. Molecular cancer 13127 (2014).

165. Khan Fa, Shukla An. Pathogenesis and treatment of gastric carcinoma: "an up-date with brief review". J Cancer Res Ther 2(4), 196-199 (2006).

166. Winkelmann J, Lin L, Schormair B et al. Mutations in DNMT1 cause autosomal dominant cerebellar ataxia, deafness and narcolepsy. Hum Mol Genet 21(10), 2205-2210 (2012). 
167. Maresca A, Zaffagnini M, Caporali L, Carelli V, Zanna C. DNA methyltransferase 1 mutations and mitochondrial pathology: is mtDNA methylated? Frontiers in Genetics 690 (2015).

168. Milutinovic S, Brown Se, Zhuang Q, Szyf M. DNA methyltransferase 1 knock down induces gene expression by a mechanism independent of DNA methylation and histone deacetylation. J Biol Chem 279(27), 27915-27927 (2004).

169. Ley $\mathrm{Tj}$, Ding L, Walter $\mathrm{Mj}$ et al. DNMT3A Mutations in Acute Myeloid Leukemia. New England Journal of Medicine 363(25), 2424-2433 (2010).

170. Fang X, Poulsen Rr, Wang-Hu J et al. Knockdown of DNA methyltransferase 3a alters gene expression and inhibits function of embryonic cardiomyocytes. FASEB journal : official publication of the Federation of American Societies for Experimental Biology 30(9), 3238-3255 (2016).

171. Gao Q, Steine Ej, Barrasa Mi et al. Deletion of the de novo DNA methyltransferase Dnmt3a promotes lung tumor progression. Proceedings of the National Academy of Sciences of the United States of America 108(44), 18061-18066 (2011).

172. Manasa P. Epigenetics-Role as Biomarker in Cancer Diagnosis. Adv Oncol Res Treat 2 r001 (2016).

173. Oshimo Y, Kuraoka K, Nakayama $\mathrm{H}$ et al. Epigenetic inactivation of SOCS-1 by CpG island hypermethylation in human gastric carcinoma. Int J Cancer 112(6), 1003-1009 (2004).

174. Zhao J, Zhao D, Poage Gm et al. Death-associated protein kinase 1 promotes growth of p53mutant cancers. J Clin Invest 125(7), 2707-2720 (2015).

175. Asada K, Nakajima T, Shimazu T et al. Demonstration of the usefulness of epigenetic cancer risk prediction by a multicentre prospective cohort study. Gut 64(3), 388-396 (2015).

** A multicenter prospective cohort study, that assessed the methylation levels of specific genes to predict the risk of metachronous gastric cancer.

176. Urakami S, Shiina H, Enokida $\mathrm{H}$ et al. Combination analysis of hypermethylated Wntantagonist family genes as a novel epigenetic biomarker panel for bladder cancer detection. Clinical cancer research : an official journal of the American Association for Cancer Research 12(7 Pt 1), 2109-2116 (2006). 
177. Hsu Hs, Chen Tp, Hung Ch et al. Characterization of a multiple epigenetic marker panel for lung cancer detection and risk assessment in plasma. Cancer 110(9), 2019-2026 (2007).

178. Koizumi W. Chemotherapy for advanced gastric cancer: review of global and Japanese status. Gastrointest Cancer Res 1(5), 197-203 (2007).

179. Housman G, Byler S, Heerboth S et al. Drug resistance in cancer: an overview. Cancers (Basel) 6(3), 1769-1792 (2014).

180. Mutze K, Langer R, Schumacher F et al. DNA methyltransferase 1 as a predictive biomarker and potential therapeutic target for chemotherapy in gastric cancer. Eur J Cancer 47(12), 18171825 (2011).

181. Lee Kd, Pai My, Hsu Cc et al. Targeted Casp8AP2 methylation increases drug resistance in mesenchymal stem cells and cancer cells. Biochem Biophys Res Commun 422(4), 578-585 (2012).

182. Maeda $\mathrm{O}$, Ando $\mathrm{T}$, Ohmiya $\mathrm{N}$ et al. Alteration of gene expression and DNA methylation in drug-resistant gastric cancer. Oncol Rep 31(4), 1883-1890 (2014).

183. Moertel Cg, Schutt Aj, Reitemeier Rj, Hahn Rg. Phase II study of 5-azacytidine (NSC-102816) in the treatment of advanced gastrointestinal cancer. Cancer Chemother Rep 56(5), 649-652 (1972).

184. Schneider Bj, Shah Ma, Klute K et al. Phase I Study of Epigenetic Priming with Azacitidine Prior to Standard Neoadjuvant Chemotherapy for Patients with Resectable Gastric and Esophageal Adenocarcinoma: Evidence of Tumor Hypomethylation as an Indicator of Major Histopathologic Response. Clin Cancer Res 23(11), 2673-2680 (2017).

185. Duenas-Gonzalez A, Medina-Franco Jl, Chavez-Blanco A, Dominguez-Gomez G, FernandezDe Gortari E. Developmental DNA methyltransferase inhibitors in the treatment of gynecologic cancers. Expert Opin Pharmacother 17(3), 323-338 (2016).

186. Tan W, Zhou W, Yu Hg, Luo Hs, Shen L. The DNA methyltransferase inhibitor zebularine induces mitochondria-mediated apoptosis in gastric cancer cells in vitro and in vivo. Biochem Biophys Res Commun 430(1), 250-255 (2013). 
187. Wu Fl, Li Rt, Yang M et al. Gelatinases-stimuli nanoparticles encapsulating 5-fluorouridine and 5-aza-2'-deoxycytidine enhance the sensitivity of gastric cancer cells to chemical therapeutics. Cancer Lett 363(1), 7-16 (2015).

188. Zhang X, Yashiro M, Ohira M, Ren J, Hirakawa K. Synergic antiproliferative effect of DNA methyltransferase inhibitor in combination with anticancer drugs in gastric carcinoma. Cancer Sci 97(9), 938-944 (2006).

189. Li Yc, Wang Y, Li Dd, Zhang Y, Zhao Tc, Li Cf. Procaine is a specific DNA methylation inhibitor with anti-tumor effect for human gastric cancer. J Cell Biochem 119(2), 2440-2449 (2018).

190. Erdmann A, Menon Y, Gros C et al. Identification and optimization of hydrazone-gallate derivatives as specific inhibitors of DNA methyltransferase 3A. Future Med Chem 8(4), 373380 (2016).

191. Xie Q, Bai Q, Zou Ly et al. Genistein inhibits DNA methylation and increases expression of tumor suppressor genes in human breast cancer cells. Genes Chromosomes Cancer 53(5), $422-$ 431 (2014).

192. Pan W, Zhu S, Yuan M et al. MicroRNA-21 and microRNA-148a contribute to DNA hypomethylation in lupus CD4+ $\mathrm{T}$ cells by directly and indirectly targeting DNA methyltransferase 1. J Immunol 184(12), 6773-6781 (2010).

193. Li Z, Li D, Zhang G et al. Methylation-associated silencing of MicroRNA-335 contributes tumor cell invasion and migration by interacting with RASA1 in gastric cancer. Am J Cancer Res 4(6), 648-662 (2014).

194. Zuo J, Xia J, Ju F et al. MicroRNA-148a can regulate runt-related transcription factor 3 gene expression via modulation of DNA methyltransferase 1 in gastric cancer. Mol Cells 35(4), 313319 (2013).

195. Zhang G, Esteve Po, Chin Hg et al. Small RNA-mediated DNA (cytosine-5) methyltransferase 1 inhibition leads to aberrant DNA methylation. Nucleic Acids Res 43(12), 6112-6124 (2015). 
196. Ooki A, Yamashita K, Yamaguchi K, Mondal A, Nishimiya H, Watanabe M. DNA damageinducible gene, reprimo functions as a tumor suppressor and is suppressed by promoter methylation in gastric cancer. Mol Cancer Res 11(11), 1362-1374 (2013).

197. Liu J, Xie Y-S, Wang F-L, Zhang L-J, Zhang Y, Luo H-S. Cytotoxicity of 5-Aza-2'deoxycytidine against gastric cancer involves DNA damage in an ATM-P53 dependent signaling pathway and demethylation of P16(INK4A). Biomed Pharmacother 67(1), 78-87 (2013).

198. Liu J, Zhang Y, Xie Ys et al. 5-Aza-2'-deoxycytidine induces cytotoxicity in BGC-823 cells via DNA methyltransferase 1 and 3a independent of p53 status. Oncology reports 28(2), 545552 (2012).

199. Eun Jw, Kim Hs, Shen Q et al. MicroRNA-495-3p functions as a tumor suppressor by regulating multiple epigenetic modifiers in gastric carcinogenesis. J Pathol 244(1), 107-119 (2018).

200. Shi $\mathrm{H}$, Chen $\mathrm{X}$, Jiang $\mathrm{H}$ et al. miR-148a suppresses cell invasion and migration in gastric cancer by targeting DNA methyltransferase 1. Oncology Letters

201. Lin S, Lin B, Wang X et al. Silencing of ATP4B of ATPase $\mathrm{H}(+) / \mathrm{K}(+)$ Transporting Beta Subunit by Intragenic Epigenetic Alteration in Human Gastric Cancer Cells. Oncol Res 25(3), 317-329 (2017).

202. Valentini V, Cellini F. Radiotherapy in gastric cancer: a systematic review of literature and new perspectives. Expert Rev Anticancer Ther 7(10), 1379-1393 (2007).

203. Qiu H, Yashiro M, Shinto O, Matsuzaki T, Hirakawa K. DNA methyltransferase inhibitor 5aza-CdR enhances the radiosensitivity of gastric cancer cells. Cancer Sci 100(1), 181-188 (2009).

204. Huang Yh, Su J, Lei Y et al. DNA epigenome editing using CRISPR-Cas SunTag-directed DNMT3A. Genome Biol 18(1), 176 (2017).

205. Mcdonald Ji, Celik H, Rois Le et al. Reprogrammable CRISPR/Cas9-based system for inducing site-specific DNA methylation. Biology Open (2016). 
206. Vojta A, Dobrinic P, Tadic V et al. Repurposing the CRISPR-Cas9 system for targeted DNA methylation. Nucleic Acids Res 44(12), 5615-5628 (2016).

** Demonstrates that CRISPR-Cas9-based tool for specific DNA methylation can decrease the expression of target loci, suggesting a potential application of CRISPR-Cas9 strategy in locusspecific epigenetic therapeutics.

207. Liu Xs, Wu H, Ji X et al. Editing DNA Methylation in the Mammalian Genome. Cell 167(1), 233-247 e217 (2016). 